An intuitive two-fluid picture of spontaneous 2D collisionless magnetic reconnection and whistler wave generation

Young Dae Yoon, and Paul M. Bellan

Citation: Physics of Plasmas 25, 055704 (2018); doi: 10.1063/1.5016345

View online: https://doi.org/10.1063/1.5016345

View Table of Contents: http://aip.scitation.org/toc/php/25/5

Published by the American Institute of Physics 


\title{
An intuitive two-fluid picture of spontaneous 2D collisionless magnetic reconnection and whistler wave generation
}

\author{
Young Dae Yoon ${ }^{\text {a) }}$ and Paul M. Bellan \\ Applied Physics and Materials Science, Caltech, Pasadena, California 91125, USA
}

(Received 17 November 2017; accepted 21 January 2018; published online 9 March 2018)

\begin{abstract}
An intuitive and physical two-fluid picture of spontaneous 2D collisionless magnetic reconnection and whistler wave generation is presented in the framework of 3D electron-magnetohydrodynamics. In this regime, canonical circulation $\left(\mathbf{Q}=m_{e} \nabla \times \mathbf{u}+q_{e} \mathbf{B}\right)$ flux tubes can be defined in analogy to magnetic flux tubes in ideal magnetohydrodynamics. Following the $3 \mathrm{D}$ behavior of these $\mathbf{Q}$ flux tubes provides a new perspective on collisionless reconnection - a perspective that has been hard to perceive via examinations of $2 \mathrm{D}$ projections. This shows that even in a 2D geometry with an ignorable coordinate, a 3D examination is essential for a full comprehension of the process. Intuitive answers are given to three main questions in collisionless reconnection: why is reconnection spontaneous, why do particles accelerate extremely fast, and why are whistler waves generated? Possible extensions to other regimes are discussed. Published by AIP Publishing. https://doi.org/10.1063/1.5016345
\end{abstract}

\section{INTRODUCTION}

Magnetic reconnection is a process in which opposing magnetic field lines come together, annihilate, reconnect, and release their stored magnetic potential energy as other forms of energy, such as kinetic, thermal and/or wave energies. This process is observed in a variety of space and astrophysical situations-solar flares, coronal mass ejections, the Earth's magnetopause and magnetotail, etc. ${ }^{1-5}$ It is also frequent in laboratory plasmas, such as tokamaks, spheromaks, and reversed-field pinches. ${ }^{6-10}$ Since reconnection acts to lower the magnetic energy in a plasma while nearly preserving magnetic helicity, it is an important part of relaxation processes that lead to equilibrium states with minimum magnetic energy.

Although magnetic reconnection is a crucial element of plasma physics, it is forbidden in ideal magneto-hydrodynamics (MHD). A typical reconnection geometry involves two counterpropagating inflows that stagnate where they meet, but because magnetic field lines are frozen into the flow, there is no means by which the field lines can escape from the stagnated flow and annihilate. Therefore, mechanisms that can break this ideal condition have been explored, and resistivity in particular, was first considered as a possible mechanism. ${ }^{11,12}$ However, this model was erroneous in many of its predictions; the reconnection rate in particular, was predicted to be too slow to explain the observed reconnection phenomena.

It is now generally agreed that collisionless terms in the generalized Ohm's law such as the Hall term and the electron inertia term are the dominant non-ideal mechanisms for fast reconnection, especially in hot plasmas. These terms are negligible on the macro-scale, but they become significant on small scales such as the ion skin depth or the ion gyroradius. Since, however, most reconnection environments are on the macro-scale, there has been much effort in finding the

Note: Paper UI3 5, Bull. Am. Phys. Soc. 62, 354 (2017).

${ }^{a}$ Invited speaker. mechanisms that bridge the two regimes, such as the plasmoid instability ${ }^{13}$ and plasma turbulence. ${ }^{14}$ In addition, a recent experiment showed that an initially MHD-driven jet can be subject to a kink-driven Rayleigh-Taylor instability, ${ }^{15}$ which effectively narrows the plasma current channel to be smaller than the ion skin depth, and that a fast reconnection event consequently occurs. ${ }^{9,10}$ Collisionless effects were evident from the emission of circularly polarized whistler waves.

In an effort to theoretically explain this event, Bellan ${ }^{16}$ analytically showed that $2 \mathrm{D}$ collisionless reconnection is a purely growing instability by doing an eigenvalue analysis. Yoon and Bellan ${ }^{17}$ expanded on this work and gave a generalized picture of spontaneous collisionless reconnection by following the behavior of canonical circulation flux tubes ${ }^{7,18,19}$ in three-dimensions.

This paper aims to both summarize and expand on Yoon and Bellan ${ }^{17}$-and the corresponding invited talk at the American Physical Society, Division of Plasma Physics meeting in Milwaukee, Wisconsin in 2017-by giving intuitive and physical answers to important questions in collisionless magnetic reconnection. Another goal of the paper is to show that even in a two-dimensional geometry with an ignorable coordinate, a three-dimensional interpretation is vital for the correct understanding of a phenomenon. The paper is organized as follows: Sec. II explains the assumptions and the reconnection model used. Section III presents the main results by not only comparing them to previous studies, but also emphasizing a new perspective on the phenomenon. Sections II and III effectively summarize the main results from Yoon and Bellan. ${ }^{17}$ Then, as an extension to this previous work, Sec. IV poses some of the important questions in reconnection and gives intuitive answers to each of them. Section V proposes some possible extensions and/or remedies for other regimes (e.g., resistive, compressible, ionsignificant), because, for the sake of simplicity, an ideal regime will be assumed in this paper. Section VI summarizes the paper. 


\section{RECONNECTION MODEL}

Because the relevant scales are smaller than the ion skin depth, ions are assumed to be stationary, so the electrons provide all the current. Incompressibility is also assumed, which is valid for $\omega_{c e}<\omega_{p e}$. The Hall and the electron inertia terms in the generalized Ohm's law are retained; the former becomes important on ion skin depth scales, and the latter on electron skin depth scales. Then, we have the following equations:

$$
\begin{gathered}
\nabla \times \mathbf{E}=-\frac{\partial \mathbf{B}}{\partial t}, \\
\nabla \times \mathbf{B}=-\mu_{0} n_{e} e \mathbf{u}_{e}, \\
\mathbf{E}+\mathbf{u}_{e} \times \mathbf{B}=-\frac{m_{e}}{e} \frac{D \mathbf{u}_{e}}{D t}-\frac{\nabla P}{n_{e} e} .
\end{gathered}
$$

Note that the convective derivative is retained, and the pressure is assumed to be isotropic for simplicity. Normalizing length by the electron skin depth $d_{e}$, time by $\left|\omega_{c e}\right|^{-1}$, and magnetic field by a reference field $B_{0}$ eliminates the constant coefficients in front of the variables in Eqs. (2) and (3). Taking the curl of the generalized Ohm's law, using Faraday's law to eliminate the electric field and rearranging give, in normalized quantities

$$
\frac{\partial \mathbf{Q}}{\partial t}=\nabla \times(\mathbf{u} \times \mathbf{Q})
$$

where

$$
\begin{aligned}
\mathbf{Q} & =\nabla^{2} \mathbf{B}-\mathbf{B}, \\
& =\nabla \times \mathbf{u}-\mathbf{B}, \\
& =\nabla \times \mathbf{u}-\nabla \times \mathbf{A}, \\
& =\nabla \times \mathbf{P}
\end{aligned}
$$

is the normalized canonical circulation ${ }^{7,20}$ (also known as generalized vorticity or canonical vorticity in various literature studies ${ }^{18,21}$ ), which is the curl of the normalized canonical momentum. The barotropic assumption was used so that $\nabla n_{e} \times \nabla P=0$. The subscript $e$ has been dropped since electrons are the only significant species. Equation (4) is the key equation in electron-magnetohydrodynamics (EMHD). ${ }^{22} \mathrm{By}$ comparison to the ideal plasma induction equation, $\partial \mathbf{B} / \partial t=\nabla \times(\mathbf{U} \times \mathbf{B})$, this means that the $\mathbf{Q}$ field lines are frozen into the electron flow, and that $\mathbf{Q}$ field lines are not allowed to reconnect in this regime, ${ }^{21,23,24}$ but $\mathbf{B}$ field lines are free to annihilate and reconnect by converting to $\nabla \times \mathbf{u}$ while preserving $\mathbf{Q}$ flux $^{25}$-although it will be shown later that this conversion is not the only mechanism that accelerates particles. Therefore, what has classically been referred to as the "dissipation region" or the "diffusion region" will be called the "conversion region," since the magnetic field lines are not reconnecting due to a dissipative or diffusive mechanism, but rather due to conversion into electron vorticity that preserves $\mathbf{Q}$ flux, which acts as an effective diffusion. Exact $\mathbf{Q}$ flux conservation also means that we can define $\mathbf{Q}$ flux tubes just as magnetic flux tubes are defined in ideal
MHD. In fact, it will be shown that tracking the threedimensional behavior of these flux tubes gives a much more intuitive and physical reconnection picture than examining projections in $2 \mathrm{D}$.

The reconnection was modelled as a $2 \mathrm{D}$ perturbation to a Harris-type current sheet structure ${ }^{26}$ with the background field in the form of

$$
\mathbf{B}_{0}=\tanh \left(x / L_{x}\right) \hat{y},
$$

where $L_{x}$ is the current sheet half thickness, and a small vector potential perturbation in the form of

$$
A_{z}=-\varepsilon \exp \left(-x^{2} / 2 \sigma_{x}^{2}-y^{2} / 2 \sigma_{y}^{2}\right)
$$

which corresponds to a small current disruption localized in $2 \mathrm{D} .^{27} \sigma_{x}$ and $\sigma_{y}$ correspond to the perturbation half thickness and half length, respectively, and $\varepsilon$ is an adjustable perturbation strength which was typically set to a small value, e.g., $10^{-3}$.

A full 3D, non-linear code was written to solve Eq. (4), with the details described in Yoon and Bellan. ${ }^{17}$ For the purpose of improving the presentation of the results, the code was migrated from Matlab 2014b to Python 3.6, and the grid spacing was improved; they were typically set as $(x, y, z)=(150,600,50)$, with step size $(\Delta x, \Delta t)=\left(0.2 d_{e}, 0.02\left|\omega_{c e}\right|^{-1}\right)$. For a typical simulation run, $L_{x}=\sigma_{x}=3 d_{e}$ and $\sigma_{y}=10 d_{e}$. The boundaries were set to be far from the central region of interest, and Neumann boundary conditions were used.

\section{RESULTS}

Figure 1 shows the two-dimensional $x-y$ cross sections of the time-dependent spontaneous collisionless reconnection process. As shown by the streamlines, the in-plane magnetic fields spontaneously come together at $x=0$ and reconnect, accelerating electrons to high velocities (red arrows). The quadrupole out-of-plane magnetic fields, represented by the yellow and blue colors, also come together and grow in magnitude. These results-quadrupole Hall magnetic fields and the geometry of the electron flow-are consistent with previous numerical and experimental studies of Hall-mediated collisionless reconnection. ${ }^{28-31}$

Reconnection rate was defined as the exponential growth rate of the outflow velocity. It is worth noting that this differs from the conventional definition of reconnection rate, which is the rate of increase of the reconnected magnetic flux. The velocity was indeed found to be growing exponentially, and the rate was fitted and compared with the analytically calculated rates by Bellan. ${ }^{16}$ Table I shows the comparison of the rate from the simulation $\gamma_{s}$ and the rate from the analytical calculation $\gamma_{c}$ for a number of different perturbation parameters. It can be seen that the orders of the rates agree extremely well.

Figure 2 shows the comparison of $\mathbf{B}$ and $\mathbf{Q}$ in both twoand three-dimensions. Again, the $\mathbf{B}$ field lines reconnect in a smooth fashion, creating the quadrupole out-of-plane Hall magnetic fields. However, Fig. 2(b) shows that the $\mathbf{Q}$ field lines, although indistinguishable from $\mathbf{B}$ at large scales, do not reconnect in the small conversion region (except due to 

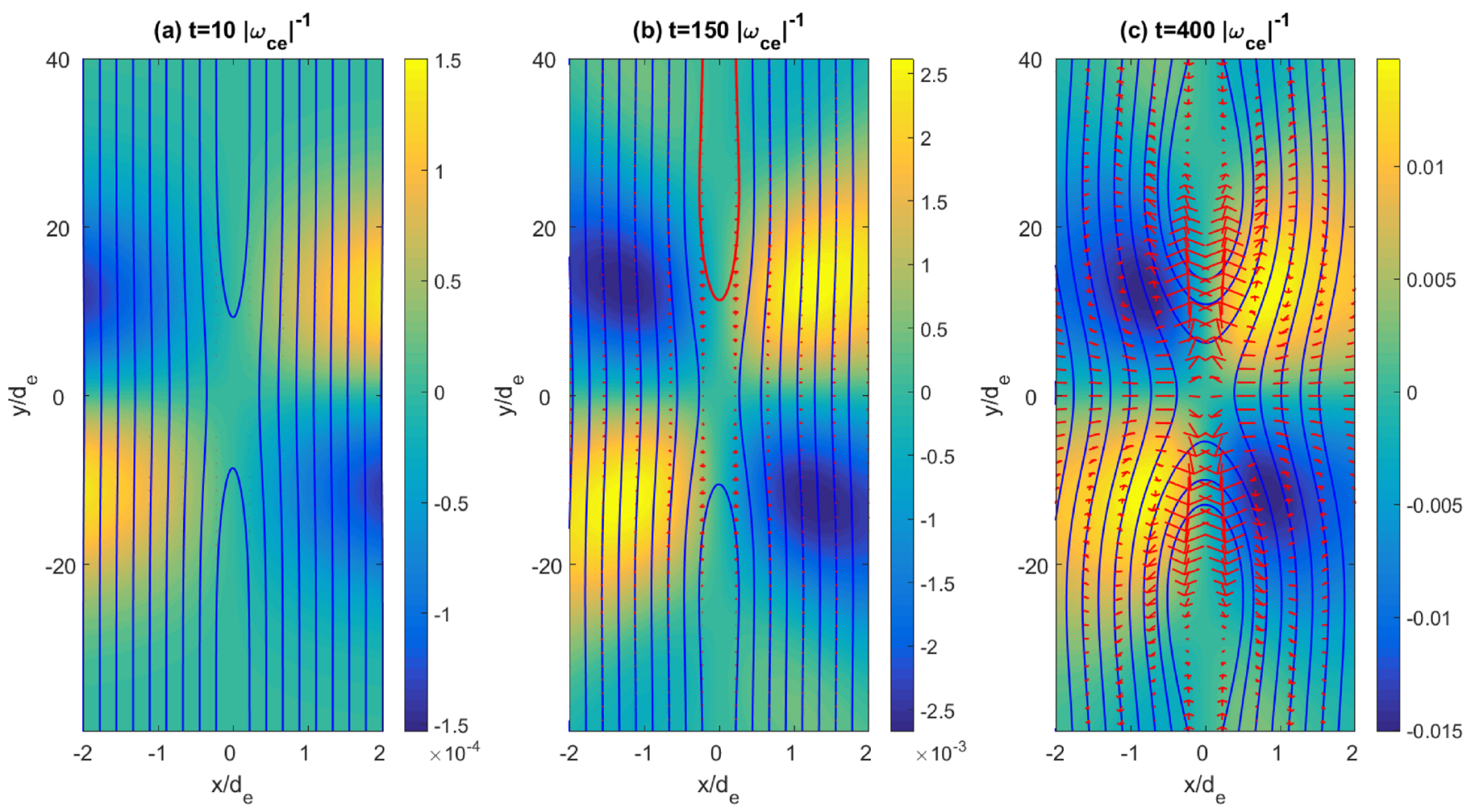

FIG. 1. Two-dimensional $x-y$ cross sections showing the time evolution of the collisionless reconnection process. The colors and the streamlines are the outof-plane and in-plane magnetic fields, respectively, and the red arrows are the electron flow vectors.

the initial perturbation and finite numerical resistivity), but only seem to pile up in 2D, as expected from Eq. (4). This phenomenon has been studied by other authors as well, ${ }^{21,23-25,32}$ where the conversion region was characterized by the pile-up of $\mathbf{Q}$ field lines and their topological invariance. However, this 2D picture raises a crucial fallacy: how can the field lines pile up when incompressibility was assumed in the first place? Clearly, there is some other process that is missing in $2 \mathrm{D}$; a full $3 \mathrm{D}$ examination is obviously necessary in order to comprehend the whole picture.

Figures 2(c) and 2(d) show the full 3D evolution of $\mathbf{B}$ and $\mathbf{Q}$, respectively. The $\mathbf{B}$ field lines convect with the central electron flow (red arrow), but only approximately. The effective magnetic diffusion can be seen to be extremely significant, since the $\mathbf{Q}$ field lines exactly convect with the electron flow in the $-z$ direction. This provides the remedy to the aforementioned $\mathbf{Q}$-pile-up fallacy; the $\mathbf{Q}$ field lines are not being piling up at all, but are "falling downwards." The electron flow can be thought of as a waterfall that carries $\mathbf{Q}$ field lines, so when seen from above $(z=+\infty)$, the $\mathbf{Q}$ lines would only appear to stagnate in the $x-y$ plane.

In the spirit of examining in full $3 \mathrm{D}$, it is useful to choose a field line [e.g., the red line in Fig. 2(d)], bunch up

TABLE I. Comparison of simulated $\gamma_{s}$ and calculated $\gamma_{c}$ for different length parameters of the current sheet.

\begin{tabular}{lccc}
\hline \hline$\sigma_{x} / d_{e}$ & $\sigma_{y} / d_{e}$ & $\gamma_{s} / \omega_{c e}\left(\times 10^{-3}\right)$ & $\gamma_{c} / \omega_{c e}\left(\times 10^{-3}\right)$ \\
\hline 1 & 10 & $40.19 \pm 0.40$ & 43.40 \\
2 & 10 & $17.56 \pm 0.10$ & 22.08 \\
2 & 16 & $12.95 \pm 0.50$ & 13.80 \\
5 & 16 & $5.21 \pm 0.05$ & 4.01 \\
\hline \hline
\end{tabular}

some field lines around it to define a flux tube, and follow the flux tube temporal behavior. Figure 3 shows the temporal evolution of a typical $\mathbf{Q}$ flux tube in 3D. Three distinct features will be important in Sec. IV and therefore merit attention: the spontaneous flux tube movement towards the $x-y$ plane conversion region and to $z \rightarrow-\infty$, the stretching and thinning of the flux tube, and its helical evolution.

\section{QUESTIONS AND ANSWERS}

\section{A. Spontaneous reconnection}

In many reconnection events, the process is spontaneous and abrupt; solar flares, ${ }^{5}$ sawtooth crashes, ${ }^{8}$ and spheromak formation $^{7}$ are some examples. Linear stability analyses show that the reconnection geometry is indeed unstable to small current perturbations, ${ }^{16,33}$ but a physical intuition for why this happens is generally lacking and so will be given as follows.

In Fig. 3(a), a small disruption to the central electron flow (red arrow) brings a segment of the $\mathbf{Q}$ flux tube closer to the center $((x, y)=(0,0)$, or the $\mathrm{x}$-point). Because the electron flow constituting the current sheet is peaked at $x=0$, this segment will be convected downwards $(-z)$ faster than other parts of the flux tube [Fig. 3(b)]. The central current is found to grow with time, forming a current singularity, ${ }^{23,24}$ so there is no restoring mechanism that brings this segment back up.

In addition, the downward motion of the flux tube generates the quadrupole out-of-plane $\mathbf{Q}$ fields [Fig. 3(c)], which are associated with the quadrupole out-of-plane $\mathbf{B}$ fields. These quadrupole $\mathbf{B}$ fields correspond to more inflow, because by Eq. (2), $u_{x} \sim-\partial B_{z} / \partial y \sim-x$ (since the out-of-plane fields go 


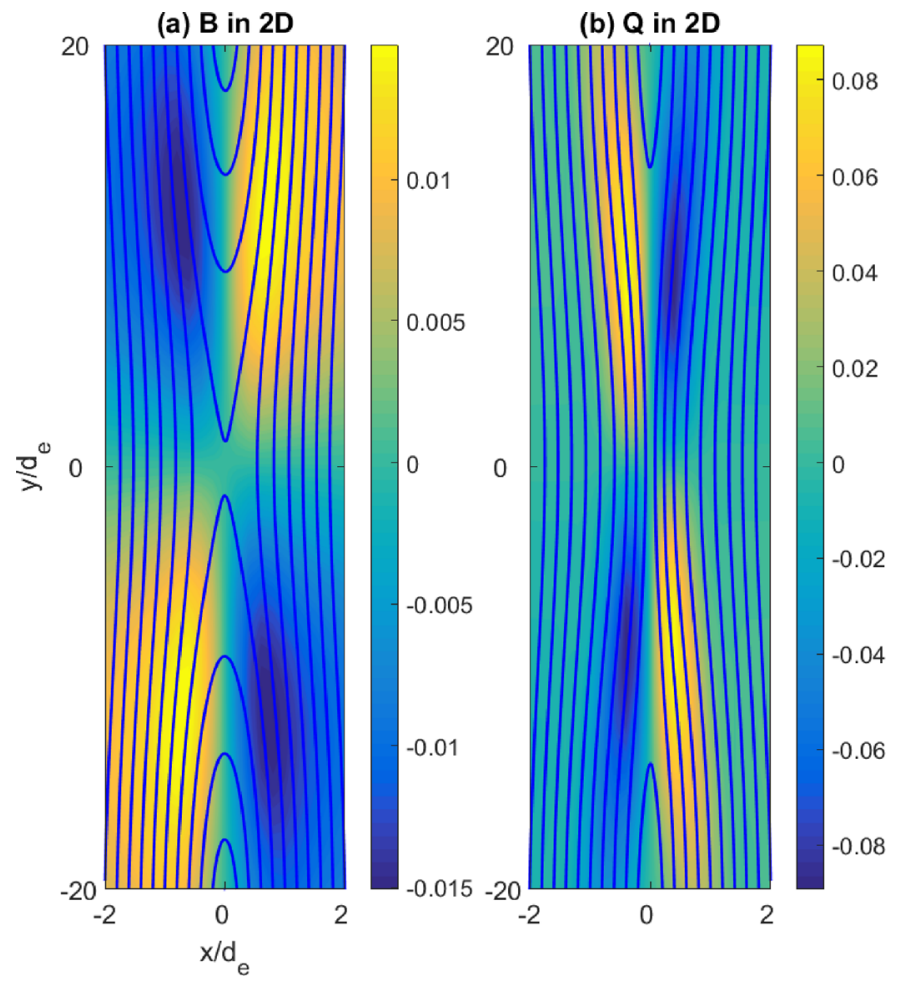

(c) B in 3D

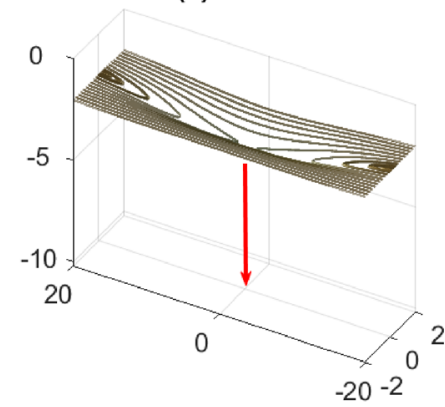

(d) $Q$ in 3D

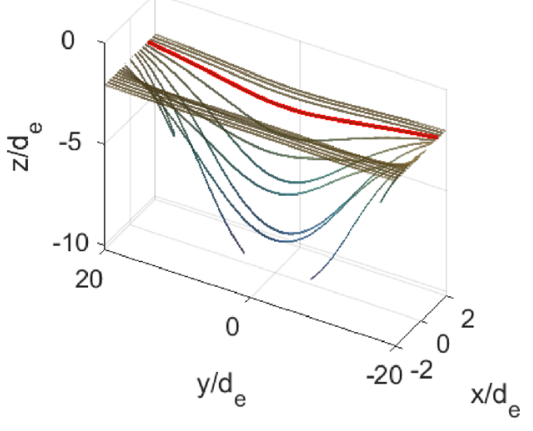

FIG. 2. 2D cross-sectional views of (a) $\mathbf{B}$ and (b) $\mathbf{Q}$. The colors and streamlines represent the out-of-plane and in-plane components, respectively. Subfigures (c) and (d) show the corresponding 3D views. The colors of the lines represent the height in $z$.

like $B_{z} \sim x y$ ). More inflow means that more $\mathbf{Q}$ field lines are brought to the center for the process to repeat.

Thus, any slight gradient of electron velocity along $\mathbf{Q}$ field lines is a sufficient condition for this instability.

\section{B. Particle acceleration}

Magnetic reconnection is also associated with particle acceleration and/or heating, as seen in ion/electron temperature increases ${ }^{10,31}$ and extreme-ultraviolet emissions, ${ }^{10}$ for example. In this regime, it is clear from the definition of $\mathbf{Q}$
[Eq. (6)] that the magnetic field can freely convert into electron vorticity. ${ }^{25}$ However, this is not the only mechanism that accelerates particles.

As in Fig. 3, the electron flow effectively pulls and stretches the $\mathbf{Q}$ flux tube, increasing its length significantly. Here, because incompressibility was assumed, the flux tube must maintain its volume, so its cross-sectional area must consequently decrease (just as a rubber band thins as it is stretched). This thinning means that the $\mathbf{Q}$ field line density-which corresponds to the magnitude of $\mathbf{Q}$-must increase, as shown by the colors of the flux tube in Fig. 3.
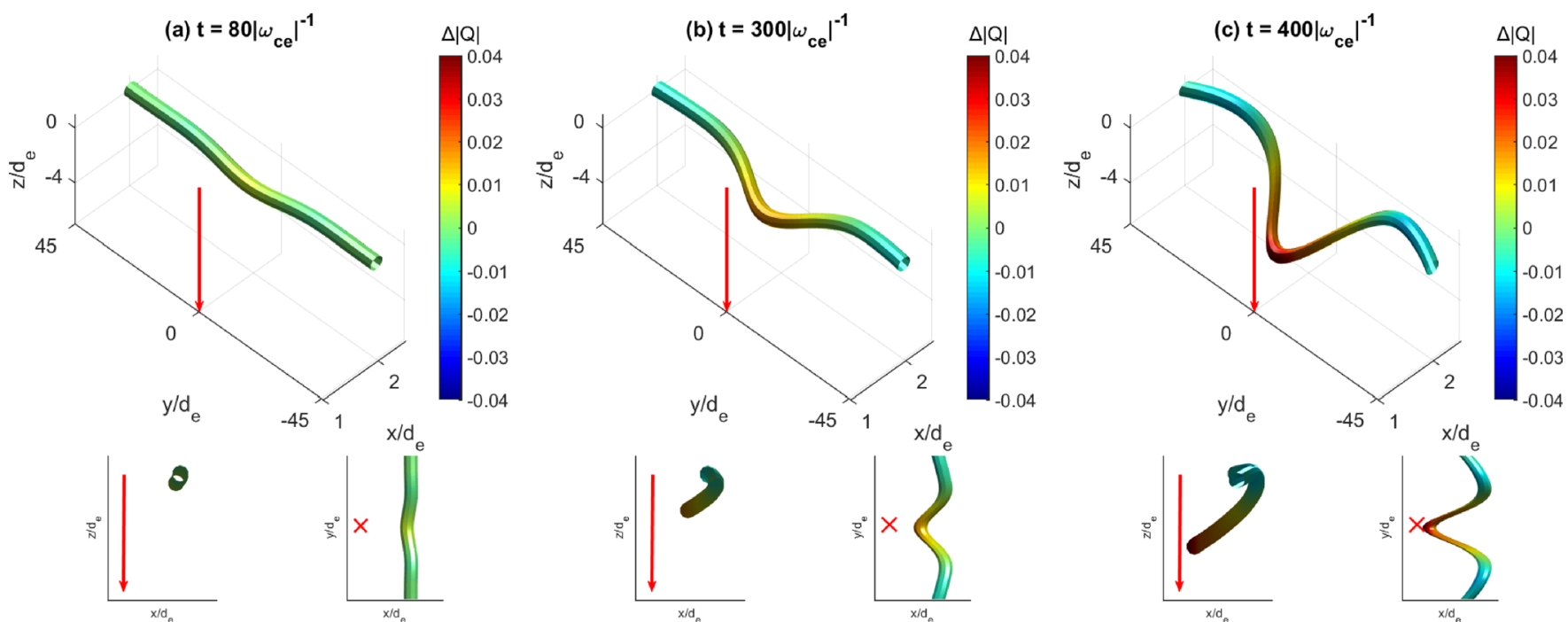

FIG. 3. Temporal evolution of a $\mathbf{Q}$ flux tube in 3D. The red arrows represent the location and direction of the electron flow at the conversion region. The colors of the tubes represent the deviation of the magnitude of $\mathbf{Q}$ from its initial value. 
Because reconnection involves cancellation of $\mathbf{B}$, amplification of $|\mathbf{Q}|$ is coincident with electron velocity increase. $|\mathbf{Q}|$ amplification can be proven to be an exponential effect, with the solution being in the following form: ${ }^{17}$

$$
\left(Q_{y}\right)_{x \approx 0, t} \approx\left(Q_{y}\right)_{x \approx 0, t=0} \exp \left(\int_{0}^{t} \frac{\partial u_{y}}{\partial y} d t^{\prime}\right),
$$

where $\partial u_{y} / \partial y \sim \partial^{2} B_{z} / \partial x \partial y$ is strictly positive since the quadrupole out-of-plane fields go like $B_{z} \sim x y$.

A simpler derivation comes from $\mathbf{Q}$ flux conservation

$$
\begin{aligned}
C & =\int \mathbf{Q} \cdot d \mathbf{S}, \\
& =\int(\nabla \times \mathbf{u}) \cdot d \mathbf{S}-\int \mathbf{B} \cdot d \mathbf{S}, \\
& =\int \mathbf{u} \cdot d \mathbf{l}-\int \mathbf{B} \cdot d \mathbf{S}
\end{aligned}
$$

so, as $\mathbf{B} \rightarrow 0$ (magnetic field cancellation) and $d \mathbf{l} \rightarrow 0$ (flux tube thinning), $\mathbf{u} \rightarrow \infty$ given that $C$ was finite to start with. The flux tube thinning, rather than the cancellation, gives the exponentially increasing effect.

Thus, extreme particle acceleration is due to a combination of interchange between the magnetic field and the electron vorticity within $\mathbf{Q} A N D \mathbf{Q}$ amplification due to flux tube thinning.

\section{Whistler wave generation}

Whistler waves have often been associated with reconnection events both in space ${ }^{4,34-38}$ and in the laboratory. ${ }^{10,39}$ There have been previous theoretical studies incorporating whistler waves into collisionless reconnection, ${ }^{28,29,40,41}$ but this paper will focus on role of the central electron current as a canonical helicity ${ }^{7,18,19,42}$ source and whistler waves as the propagator.

Figure 3 shows that a typical $\mathbf{Q}$ flux tube evolves helically over time. This is due to the central electron flow, which induces a velocity shear between the conversion region and the other regions. Mathematically, the normalized canonical helicity density $\kappa=\mathbf{P} \cdot \mathbf{Q}$ evolves in time as follows:

$$
\begin{aligned}
\frac{D \kappa}{D t} & =\mathbf{Q} \cdot \nabla\left(\frac{u^{2}}{2}-P-\mathbf{u} \cdot \mathbf{A}\right), \\
& =\mathbf{Q} \cdot \nabla \mathcal{L},
\end{aligned}
$$

where $\mathcal{L}=\frac{u^{2}}{2}-P-\mathbf{u} \cdot \mathbf{A}$ is the Lagrangian density of the system (proof in the Appendix). This is the canonical helicity conservation equation ${ }^{7,42}$ for this specific regime. The central electron flow is a source of $\nabla \mathcal{L}$ along the $\mathbf{Q}$ field lines, so it is in turn a source of canonical helicity density. Because the Fourier analysis of the linearized version of Eq. (4) gives the whistler dispersion relation, whistler waves represent a dynamic winding/unwinding of $\mathbf{Q}$ field lines just as torsional Alfvèn waves do of $\mathbf{B}$ lines. Therefore, whistler wave generation and propagation in collisionless reconnection can be seen as the central electron flow curling up the $\mathbf{Q}$ flux tubes and whistler waves propagating this helicity density in the $\pm y$ directions.

We now turn attention to the behavior of $\mathbf{B}$ field lines. Figure 1 shows that there is an additional region of out-ofplane magnetic fields that develops near the outflow regions [e.g., black box in Fig. 1(c)]. These fields, which have been previously observed by other numerical studies as well $^{28,29,41}$ but generally not discussed in three-dimensional detail, suggest wave-like behavior, so it is worth examining how a typical reconnected $\mathbf{B}$ field line [e.g., red line in Fig. 1(b)] evolves taking these fields into account.

Figure 4 shows the 3D time evolution of a typical reconnected $\mathbf{B}$ field line. We can regard the initial field line as the background field, and the final field line as the background field plus the perturbations to this field. If the initial field line
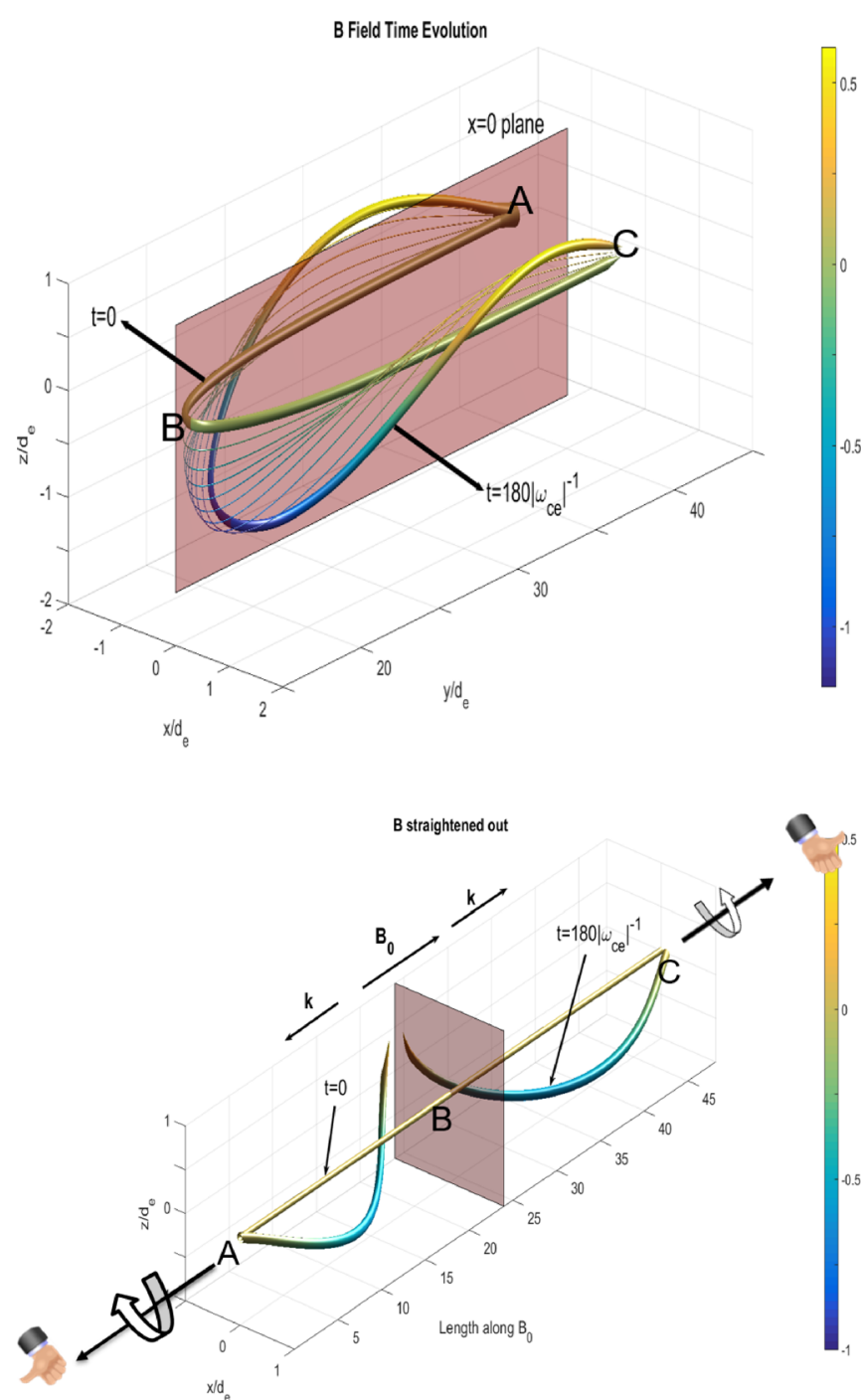

FIG. 4. Temporal evolution of one of the reconnected magnetic field lines (e.g., red line in Fig. 1). The bottom figure shows the same field lines when the initial field line is straightened out. The A, B, and Cs in both figures correspond to identical locations. The colors represent the height in $z$. Reproduced with permission from Phys. Plasmas 24(5), 052114 (2017). Copyright 2017 AIP Publishing. ${ }^{17}$ 
is stretched so that it mimics a uniform background field (as in the bottom subfigure), we can see that the spatial helicity of the final field corresponds ${ }^{43}$ to the conversion region (red rectangle) acting as the source of two whistler waves propagating away from this source in the $\pm y$ directions. This picture is consistent with recent spacecraft observations ${ }^{35,37,38}$ where the conversion region was a possible source for whistler waves towards the outflow regions.

\section{EXTENSIONS TO OTHER REGIMES}

So far in this paper, an ideal situation was assumed for simplicity. However, even in non-ideal situations, the picture of canonical flux tubes helps the comprehension of a complicated reconnection process. The following non-ideal considerations are not based on definitive results from the authors' work, but are rather suggestions for future investigations that can be done with this picture.

Finite resistivity, first of all, could be considered. For example, previous studies ${ }^{44,45}$ have shown that finite resistivity slows down the formation of current singularities. ${ }^{23,24}$ This can be explained as follows: resistivity will allow $\mathbf{Q}$ field lines to reconnect, inhibiting their apparent pile-up in the $x-y$ plane. Therefore, a $\mathbf{Q}$ singularity will not develop. A non-scalar pressure would also provide effective diffusion.

The compressible case can also be considered-in fact, there have been previous studies in this regime, ${ }^{46-48}$ and they have shown that in this case, the conserved quantity is slightly modified from pure canonical circulation flux to include density. They have also shown ${ }^{48}$ that compressibility inhibits the development of secondary instabilities (e.g., Kelvin-Helmholtz) or turbulence ${ }^{48,49}$ that arise due to the aforementioned current singularities. Therefore, compressibility, together with resisitivity, could be the key to the transition from abrupt reconnection to quasi-steady-state reconnection.

Equation (4) actually applies to any other charged species, ${ }^{7,20,42}$ such as ions, if the pressure is assumed to be scalar and barotropic, and collisions are ignored. It is also applicable in both kinetic and fluid regimes, with slightly different definitions of the terms for each regime. ${ }^{42,50}$ Therefore, it would be useful to examine, for example, how an ion canonical flux tube behaves in a regime where ion motion is important. Also, the exact mechanism with which canonical helicity density propagates over multiple scales to magnetic helicity in the macroscale might also be important in explaining the abundance of flux ropes in space.

\section{CONCLUSION}

In conclusion, an intuitive, physical description of 2D spontaneous collisionless magnetic reconnection in the twofluid EMHD regime has been presented. Because in this EMHD regime, electron canonical circulation is frozen into the electron flow, the temporal three-dimensional evolution of the canonical circulation flux tubes in the reconnection process was examined. It was shown that even in a $2 \mathrm{D}$ geometry, a 3D investigation is necessary for a full comprehension of the phenomenon.
Intuitive answers were given to three main questions. First, the reason why many reconnection events are nondriven and spontaneous is that any gradient of electron flow along canonical circulation flux tubes causes an instability. Second, the mechanism that accelerates electrons to huge velocities is a combination of interchange between the electron vorticity and the magnetic field and flux tube thinning due to pulling and stretching by the central electron flow. Finally, whistler waves are generated from collisionless reconnection events because the central electron current acts as a canonical helicity density source, and whistler waves carry this helicity density in the outflow directions.

\section{ACKNOWLEDGMENTS}

This material is based upon work supported by the National Science Foundation under Award No. 1059519, by the Air Force Office of Scientific Research under Award No. FA9550-11-1-0184, and by the U.S. Department of Energy Office of Science, Office of Fusion Energy Sciences under Award No. DE-FG02-04ER54755.

\section{APPENDIX: LAGRANGIAN DENSITY OF THE SYSTEM}

If and only if $\mathcal{L}=\frac{u^{2}}{2}-P-\mathbf{u} \cdot \mathbf{A}$ is a normalized Lagrangian density of the system, the Euler-Lagrange equation

$$
\nabla_{x} L-\frac{D}{D t} \nabla_{u} L=0,
$$

where $L(\mathbf{x}, \mathbf{u}, t)=\int \mathcal{L}(\mathbf{x}, \mathbf{u}, t) d V$ is the Lagrangian, must give the equation of motion of the system [Eq. (3)]. $\nabla_{x}$ is the spatial gradient and $\nabla_{u}$ is the gradient in velocity space. Here

$$
\begin{aligned}
\nabla_{x} L & =\int\left[-\nabla_{x} P-\nabla_{x}(\mathbf{u} \cdot \mathbf{A})\right] d V, \\
& =\int\left[-\nabla_{x} P-\mathbf{u} \cdot \nabla_{x} \mathbf{A}-\mathbf{u} \times\left(\nabla_{x} \times \mathbf{A}\right)\right] d V, \\
& =\int\left[-\nabla_{x} P-\mathbf{u} \cdot \nabla_{x} \mathbf{A}-\mathbf{u} \times \mathbf{B}\right] d V
\end{aligned}
$$

since only $P$ and $\mathbf{A}$ are explicitly spatially dependent. Also

$$
\begin{aligned}
\nabla_{u} L & =\int \nabla_{u} \mathcal{L} d V, \\
& =\int(\mathbf{u}-\mathbf{A}) d V .
\end{aligned}
$$

So

$$
\begin{aligned}
\frac{D}{D t} \nabla_{u} L & =\int \frac{\partial}{\partial t}(\mathbf{u}-\mathbf{A}) d V+\int(\mathbf{u}-\mathbf{A})(\mathbf{u} \cdot d \mathbf{s}), \\
& =\int\left(\frac{\partial \mathbf{u}}{\partial t}-\frac{\partial \mathbf{A}}{\partial t}\right) d V+\int \nabla_{x} \cdot[\mathbf{u}(\mathbf{u}-\mathbf{A})] d V, \\
& =\int\left(\frac{D \mathbf{u}}{D t}+\mathbf{E}-\mathbf{u} \cdot \nabla_{x} \mathbf{A}\right) d V .
\end{aligned}
$$

Therefore 


$$
\begin{aligned}
\nabla_{x} L-\frac{D}{D t} \nabla_{u} L & =\int\left[\begin{array}{c}
-\nabla_{x} P-\mathbf{u} \cdot \nabla_{x} \mathbf{A}-\mathbf{u} \times \mathbf{B} \\
-\frac{D \mathbf{u}}{D t}-\mathbf{E}+\mathbf{u} \cdot \nabla_{x} \mathbf{A}
\end{array}\right] d V \\
& =\int\left[-\nabla_{x} P-\mathbf{u} \times \mathbf{B}-\frac{D \mathbf{u}}{D t}-\mathbf{E}\right] d V \\
& =0 .
\end{aligned}
$$

Setting the integrand to zero gives us the original normalized equation of motion, or the generalized Ohm's law [Eq. (3)]

$$
\frac{D \mathbf{u}}{D t}=-\mathbf{E}-\mathbf{u} \times \mathbf{B}-\nabla P
$$

\section{Q.E.D.}

${ }^{1}$ E. N. Parker, Cosmical Magnetic Fields: Their Origin and Their Activity (Oxford University Press, 1979).

${ }^{2}$ R. M. Kulsrud, Phys. Plasmas 5, 1599 (1998).

${ }^{3}$ E. Priest and T. Forbes, Magnetic Reconnection (Cambridge University Press, 2000).

${ }^{4}$ X. H. Deng and H. Matsumoto, Nature 410, 557 (2001).

${ }^{5}$ T. G. Forbes, Nat. Phys. 9, 456 (2013).

${ }^{6}$ M. Yamada, Phys. Plasmas 1, 3269 (1994).

${ }^{7}$ P. M. Bellan, Spheromaks (Imperial College Press, 2000).

${ }^{8}$ J. A. Wesson, Nucl. Fusion 30, 2545 (1990).

${ }^{9}$ A. Moser, Nature 482, 379 (2012).

${ }^{10}$ K.-B. Chai, X. Zhai, and P. M. Bellan, Phys. Plasmas 23, 032122 (2016).

${ }^{11}$ P. Sweet, Electromagnetic Phenomena in Cosmical Physics (Cambridge University Press, 1958)

${ }^{12}$ E. N. Parker, Astrophys. J. Suppl. Ser. 8, 177 (1963).

${ }^{13}$ A. Bhattacharjee, Y.-M. Huang, H. Yang, and B. Rogers, Phys. Plasmas 16, 112102 (2009).

${ }^{14}$ A. Retinò, D. Sundkvist, A. Vaivads, F. Mozer, M. André, and C. Owen, Nat. Phys. 3, 235 (2007).

${ }^{15}$ X. Zhai and P. M. Bellan, Phys. Plasmas 23, 032121 (2016).

${ }^{16}$ P. M. Bellan, Phys. Plasmas 21, 102108 (2014).

${ }^{17}$ Y. D. Yoon and P. M. Bellan, Phys. Plasmas 24, 052114 (2017).

${ }^{18}$ S. You, Phys. Plasmas 19, 092107 (2012).

${ }^{19}$ S. You, Plasma Phys. Controlled Fusion 56, 064007 (2014).

${ }^{20}$ P. Bellan, Phys. Fluids B: Plasma Phys. 5, 1955 (1993).

${ }^{21}$ S. Bulanov, F. Pegoraro, and A. Sakharov, Phys. Fluids B: Plasma Phys. 4, 2499 (1992).

${ }^{22}$ A. Kingsep, K. Chukbar, and V. Yan'kov, Reviews of Plasma Physics, edited by B. Kadomtsev (Consultants Bureau, New York, 1990), Vol. 16, p. 143.

${ }^{23}$ M. Ottaviani and F. Porcelli, Phys. Rev. Lett. 71, 3802 (1993).

${ }^{24}$ D. Biskamp, E. Schwarz, and J. F. Drake, Phys. Plasmas 4, 1002 (1997).
${ }^{25}$ E. Cafaro, D. Grasso, F. Pegoraro, F. Porcelli, and A. Saluzzi, Phys. Rev. Lett. 80, 4430 (1998).

${ }^{26}$ E. G. Harris, Il Nuovo Cimento (1955-1965) 23, 115 (1962).

${ }^{27}$ P. M. Bellan, Phys. Plasmas 5, 3081 (1998).

${ }^{28}$ J. F. Drake, M. A. Shay, and M. Swisdak, Phys. Plasmas 15, 042306 (2008).

${ }^{29}$ K. Fujimoto and R. D. Sydora, Geophys. Res. Lett. 35, 19, https://doi.org/ 10.1029/2008GL035201 (2008).

${ }^{30}$ Y. Ren, M. Yamada, S. Gerhardt, H. Ji, R. Kulsrud, and A. Kuritsyn, Phys. Rev. Lett. 95, 055003 (2005).

${ }^{31}$ M. Yamada, J. Yoo, J. Jara-Almonte, H. Ji, R. M. Kulsrud, and C. E. Myers, Nat. Commun. 5, 4774 (2014).

${ }^{32}$ D. Grasso, F. Califano, F. Pegoraro, and F. Porcelli, Phys. Rev. Lett. 86, 5051 (2001).

${ }^{33}$ N. Attico, F. Califano, and F. Pegoraro, Phys. Plasmas 7, 2381 (2000).

${ }^{34}$ X. Wei, J. Cao, G. Zhou, O. Santolík, H. Reme, I. Dandouras, N. Cornilleau-Wehrlin, E. Lucek, C. Carr, and A. Fazakerley, J. Geophys. Res.: Space Phys. 112, A10, https://doi.org/10.1029/2006JA011771 (2007).

${ }^{35}$ S. Y. Huang, H. S. Fu, Z. G. Yuan, A. Vaivads, Y. V. Khotyaintsev, A. Retino, M. Zhou, D. B. Graham, K. Fujimoto, F. Sahraoui, X. H. Deng, B. Ni, Y. Pang, S. Fu, D. D. Wang, and X. Zhou, J. Geophys. Res.: Space Phys. 121, 6639, https://doi.org/10.1002/2016JA022650 (2016).

${ }^{36}$ D. B. Graham, A. Vaivads, Y. V. Khotyaintsev, and M. Andr, J. Geophys. Res.: Space Phys. 121, 1934, https://doi.org/10.1002/2015JA021239 (2016).

${ }^{37}$ O. L. Contel, A. Retinó, H. Breuillard, L. Mirioni, P. Robert, A. Chasapis, B. Lavraud, T. Chust, L. Rezeau, F. D. Wilder, D. B. Graham, M. R. Argall, D. J. Gershman, P.-A. Lindqvist, Y. V. Khotyaintsev, G. Marklund, R. E. Ergun, K. A. Goodrich, J. L. Burch, R. B. Torbert, J. Needell, M. Chutter, D. Rau, I. Dors, C. T. Russell, W. Magnes, R. J. Strangeway, K. R. Bromund, H. K. Leinweber, F. Plaschke, D. Fischer, B. J. Anderson, G. Le, T. E. Moore, C. J. Pollock, B. L. Giles, J. C. Dorelli, L. Avanov, and Y. Saito, Geophys. Res. Lett. 43, 5943, https://doi.org/ 10.1002/2016GL068968 (2016).

${ }^{38}$ X. Tang, C. Cattell, J. Dombeck, L. Dai, L. B. Wilson, A. Breneman, and A. Hupach, Geophys. Res. Lett. 40, 2884, https://doi.org/10.1002/ grl.50565 (2013).

${ }^{39}$ H. Ji, S. Terry, M. Yamada, R. Kulsrud, A. Kuritsyn, and Y. Ren, Phys. Rev. Lett. 92, 115001 (2004)

${ }^{40}$ M. E. Mandt, R. E. Denton, and J. F. Drake, Geophys. Res. Lett. 21, 73, https://doi.org/10.1029/93GL03382 (1994).

${ }^{41}$ N. Jain and A. S. Sharma, Phys. Plasmas 22, 102110 (2015).

${ }^{42}$ S. You, Phys. Plasmas 23, 072108 (2016).

${ }^{43}$ R. L. Stenzel and J. M. Urrutia, Phys. Rev. Lett. 114, 205005 (2015).

${ }^{44}$ M. Ottaviani and F. Porcelli, Phys. Plasmas 2, 4104 (1995).

${ }^{45}$ A. Bhattacharjee, K. Germaschewski, and C. Ng, Phys. Plasmas 12, 042305 (2005).

${ }^{46}$ B. N. Kuvshinov, E. Westerhof, T. J. Schep, and M. Berning, Phys. Lett. A 241, 287 (1998)

${ }^{47}$ N. Attico, F. Califano, and F. Pegoraro, Phys. Plasmas 8, 16 (2001).

${ }^{48}$ D. Del Sarto, F. Califano, and F. Pegoraro, Phys. Plasmas 12, 012317 (2005).

${ }^{49}$ J. Drake, D. Biskamp, and A. Zeiler, Geophys. Res. Lett. 24, 2921, https:// doi.org/10.1029/97GL52961 (1997).

${ }^{50}$ J. von der Linden, "Investigating the dynamics of canonical flux tubes," Ph.D. thesis (University of Washington, 2017). 